\title{
A newly recorded Rickettsia of the Torix group is a recent intruder and an endosymbiont in the whitefly Bemisia tabaci
}

\author{
Hua-Ling Wang $\mathbb{D}^{1},{ }^{1,2 \dagger}$ Teng Lei, ${ }^{1 \dagger}$ Xiao-Wei Wang, ${ }^{1}$ \\ M. N. Maruthi, ${ }^{2}$ Dan-Tong Zhu, ${ }^{1}$ Stephen L. Cameron, ${ }^{3}$ \\ Qiong Rao, ${ }^{4}$ Hong-Wei Shan, ${ }^{1}$ John Colvin, ${ }^{2}$ \\ Yin-Quan Liu (D) ${ }^{1}$ and Shu-Sheng Liu ${ }^{1 *}$ \\ ${ }^{1}$ The Ministry of Agriculture Key Laboratory of Molecular \\ Biology of Crop Pathogens and Insects, Institute of \\ Insect Sciences, Zhejiang University, 866 Yuhangtang \\ Road, Hangzhou, 310058, China. \\ ${ }^{2}$ Natural Resources Institute, University of Greenwich, \\ Kent, ME4 4TB, UK. \\ ${ }^{3}$ Department of Entomology, Purdue University, 901 \\ West State Street, West Lafayette, IN, 479074, USA. \\ ${ }^{4}$ School of Agriculture and Food Science, Zhejiang A \& \\ F University, Lin'an, 311300, Zhejiang, China.
}

\section{Summary}

The bacterium Rickettsia is found widely in phytopha-gous insects and often exerts profound effects on the phenotype and fitness of its hosts. Here, we decrypt a new, independent, phylogenetically ancient Torix Rick-ettsia endosymbiont found constantly in a laboratory line of an economically important insect Asia II 7, a putative species of the Bemisia tabaci whitefly complex (Hemiptera: Aleyrodidae), and occasionally in field whitefly populations. This new Rickettsia distributes throughout the body of its whitefly host. Genetically, compared to Rickettsia_bellii_MEAM1 found earlier in whiteflies, the new Rickettsia species has more gene families and pathways, which may be important factors in shaping specific symbiotic relationships. We propose the name 'Candidatus Rickettsia_Torix_Bemisia_tabaci

(RiTBt)' for this new endosymbiont associated with whiteflies. Comparative genomic analyses indicate that RiTBi may be a relatively recent intruder in whiteflies given its low abundance in the field and relatively larger genome compared to Rickettsia_bellii_MEAM1.

\section{Introduction}

Plant sap-sucking insects, such as psyllids, aphids, and mealybugs, are members of the Hemiptera sub-order Sternorrhyncha and have obligatory as well as facultative associations with a diverse range of prokaryotic endosymbionts (Baumann, 2005). Many studies have shown the vital roles of various bacteria in the biology of their hosts, such as synthesis of essential amino acids (Douglas, 2006), tolerance to high temperatures (Montllor et al., 2002), and facilitating virus transmission (Gottlieb et al., 2010).

The whitefly Bemisia tabaci (Gennadius) (Hemiptera: Aleyrodidae) is a cryptic species complex with at least 36 putative species (Liu et al., 2012; Boykin, 2014). They are distributed mostly in the tropics and subtropics, and some of them, such as Mediterranean (MED), Middle East-Asia Minor 1 (MEAM1), Sub-Saharan Africa 1 (SSA1) and Asia II 1, are important agricultural pests (Liu et al., 2007). Whiteflies of the B. tabaci species complex share a long-term and intimate association with bacterial symbionts. So far, one obligate endosymbiont, 'Candidatus Portiera aleyrodidarum' (Oceanospirillales), (hereafter Portiera), has been recognized and is localized in the bacteriome, a tissue composed of bacteriocytes (Zchori-Fein and Brown, 2002). Portiera is essential to the synthesis of essential amino acids and carotenoids for its hosts and is transmitted vertically via the female ovary (Gottlieb et al., 2006; Luan et al., 2016). Seven genera of facultative endosymbionts have been found, including Arsenophonus spp. (Enterobacteriales) (Zchori-Fein and Brown, 2002), 'Candidatus Cardinium hertigii' (Bacteroidales) (Weeks et al., 2003), 'Candidatus Fritschea bemisiae' (Chlamydiales) (Everett et al., 2005), 'Candidatus Hamiltonella defensa' (Enterobacteriales) (Zchori-Fein and Brown, 2002), Hemipteriphilus asiaticus/OLO (Rickettsiales) (Bing et al., 2013), Wolbachia spp. (Rickettsiales) (Nirgianaki et al., 2003), and Rickettsia spp. (Rickettsiales)(Gottlieb et al., 2006). Although many facultative endosymbionts have been found, the functional properties of most of them are barely known. 
One of these facultative endosymbionts, Rickettsia (class: Alpha-proteobacteria; order: Rickettsiales) contains a wide diversity of species. Phylogenomic analyses indicate the Rickettsia can be divided into at least four groups: ancestral group (AG), typhus group (TG), transitional group (TRG), and spotted fever group (SFG) (Gillespie et al., 2008). Based on partial sequence analysis, Weinert et al. (2009) suggested further delineations of the genus into 12 groups: Hydra, Tundra, Torix, Rhizobius, Meloidae, Bellii, Onychiurus, Adalia, Canadensis, Spotted Fever, Typhus, and Transitional, with Orientia tsutsugamushi as the outgroup. Up to now, over 60 Rickettsia genomes have been published from a variety of host systems. Most of these belong to Bellii, Canadensis, Spotted Fever, Typhus, and Transitional group, with only one to the Torix group (Pilgrim et al., 2017).

A notable characterization of Rickettsia is that some species are pathogenic to vertebrates (Weinert et al., 2009), such as $R$. prowazekii, $R$. rickettsii, and $R$. conorii. Rickettsia have also been detected in a wide variety of invertebrates and have profound effects on the biology of the hosts and vector-pathogen interactions (Pilgrim et al., 2017). As for B. tabaci, only Rickettsia_bellii_MEAM1 has been studied in some detail. Two localization patterns in the hosts have been recognized: 'scattered', where the bacterium distributes throughout the whitefly hemocoel, excluding the bacteriocytes; and 'confined', where the bacterium is restricted to the bacteriocytes (Caspi-Fluger et al., 2011). Rickettsia_bellii_MEAM1 with the 'scattered' distribution pattern in MEAM1 is transmitted horizontally through plants (Caspi-Fluger et al., 2012) and can enhance heat tolerance of the host (Brumin et al., 2011), increase fecundity (Himler et al., 2011), and promote virus transmission (Ghanim, 2016). Collectively, these properties make Rickettsia biologically significant for its host, yet so far, the other relevant Rickettsia species has been largely understudied.

Here, we firstly describe a novel Torix Rickettsia species that has a symbiotic relationship with species Asia II 7 of the B. tabaci whitefly complex. A PacBio 16S screening project identified a new 16S rRNA allied to a novel Rickettsia and its association with a specific host. We then investigated several aspects of the biology of this new Rickettsia including its field distribution, host range, in vivo localization, taxonomic position, and genomic properties. Finally, we sought to compare this new Rickettsia symbiont with the different Rickettsia previously described in $B$. tabaci. The results showed species-level divergence between this new Rickettsia and other Rickettsia recorded previously. We, thus, propose the name 'Candidatus Rickettsia_Torix_ Bemisia_tabaci (RiTBt)' for this new species of Rickettsia. Our analysis adds new insights into microbial community residing in the $B$. tabaci species complex and their diverse interactions with hosts.

\section{Results}

Discovery of RiTBt

In a comprehensive screening for facultative endosymbionts of the B. tabaci whitefly complex using PacBio 16S, a Rickettsia-like bacterial 16S rRNA gene was consistently obtained from a laboratory colony of Asia II 7. Bayesian analysis based on the $16 S$ rRNA gene showed that this new Rickettsia was not clustered with the previously identified Rickettsia_bellii_MEAM1 but rather branched between OLO_Bemisia-China1_JX042443 (Hemipteriphilus asiaticus from $B$. tabaci China 1) and the remaining Rickettsia spp. (Fig. 1.A). Additional analyses of the proteincoding genes glt $A$ and groEL yielded similar phylogenetic relationships (Fig. 1.B and $C$ ). The nucleotide sequence identities of $16 S$ rRNA, gltA, and groEL genes between RiTBt and Rickettsia_bellii_MEAM1 are 96\% (1442 bp), $78 \%$ (481 bp) and 78\% (1646 bp) respectively. Based on these results, we propose that the new Rickettsia is a new species residing in $B$. tabaci and named it as 'Candidatus Rickettsia_Torix_Bemisia_tabaci' (RiTBt).

Prevalence of RiTBt in the field and a laboratory whitefly population

To examine the geographical distribution of RiTBt and its association with various species of the B. tabaci complex, we screened 512 adults that were collected from major crop plants and vegetables such as cotton, sweet potato, pumpkin, eggplant, cucumber, and tomato, in 22 locations of 14 provinces in China (Fig. 2. and Supporting Information Table S1). As B. tabaci is a cryptic species complex, and more than one species may occur on the same host plant within a field block, we firstly established the species status of whiteflies by individual mtCOI barcoding before running the diagnostic PCR for RiTBt. Collected specimens were assigned to eight $B$. tabaci cryptic species: MED, MEAM1, China 1, China 2, Asia I, Asia II 1, Asia II 6 and Asia II 7 (Fig. 2. and Supporting Information Table S1). Five of the 512 adults examined were RiTBt-positive and were all Asia II 1 collected from plants of Ipomoea batatas and Brassica chinensis in Zhaoqing, Guangdong province (Fig. 2. and Supporting Information Table S1). In contrast, in an Asia II 7 laboratory population, all individuals tested were infected with RiTBt (46 of $46,100 \%$ ). We then conducted the test consecutively for five generations of the whitefly host, with 30 adults in each generation, and found that all individuals tested in each of the generations were infected with RiTBt. Of the eight screened 


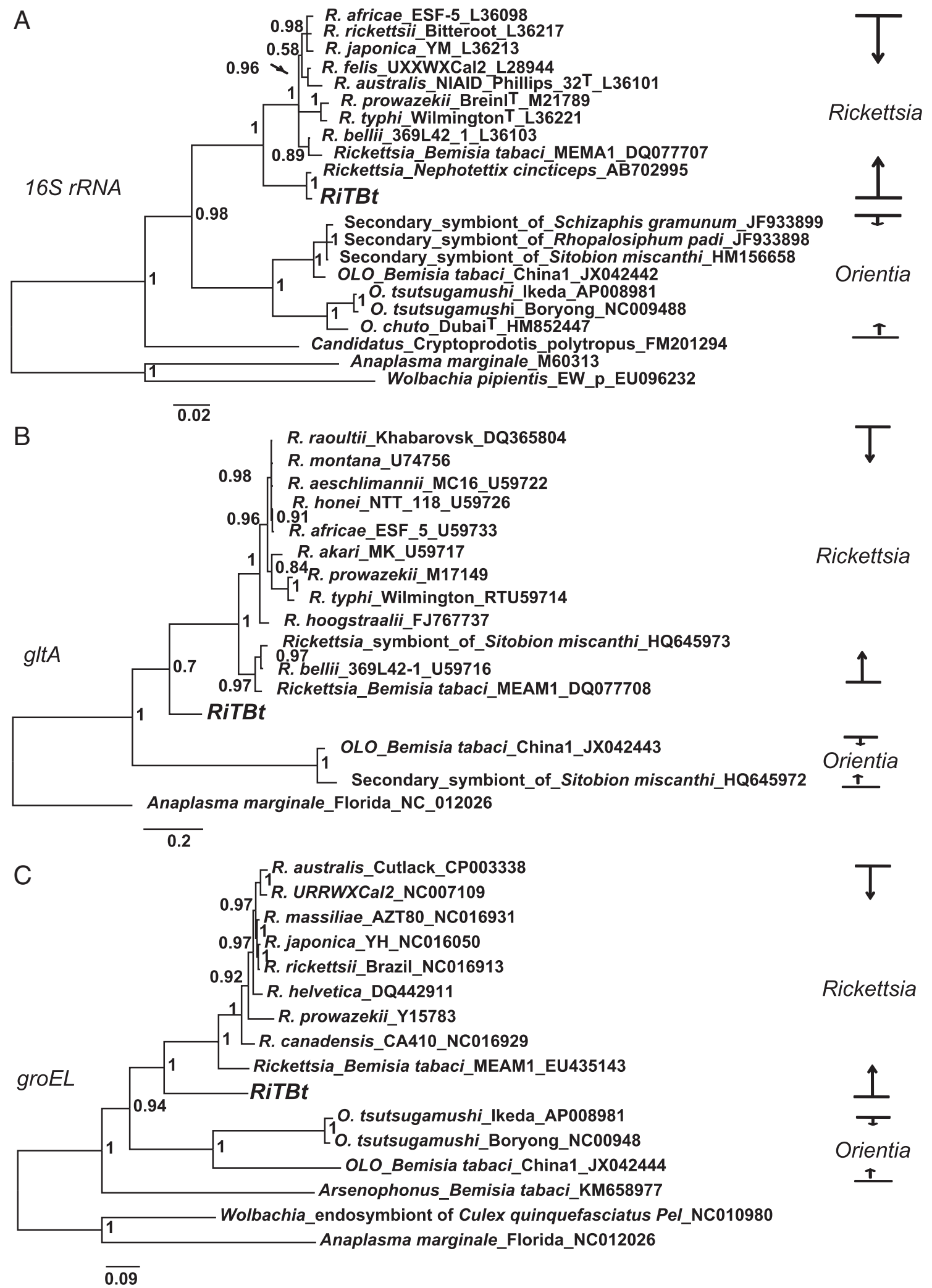

Fig. 1. Phylogenetic relationship of RiTBt with other Rickettsia based on bacterial $16 S r R N A(A) / g / t A(B) / g r o E L(C)$ gene sequences. Posterior probabilities for the Bayesian phylogeny at $50 \%$ or higher are shown at the nodes. 


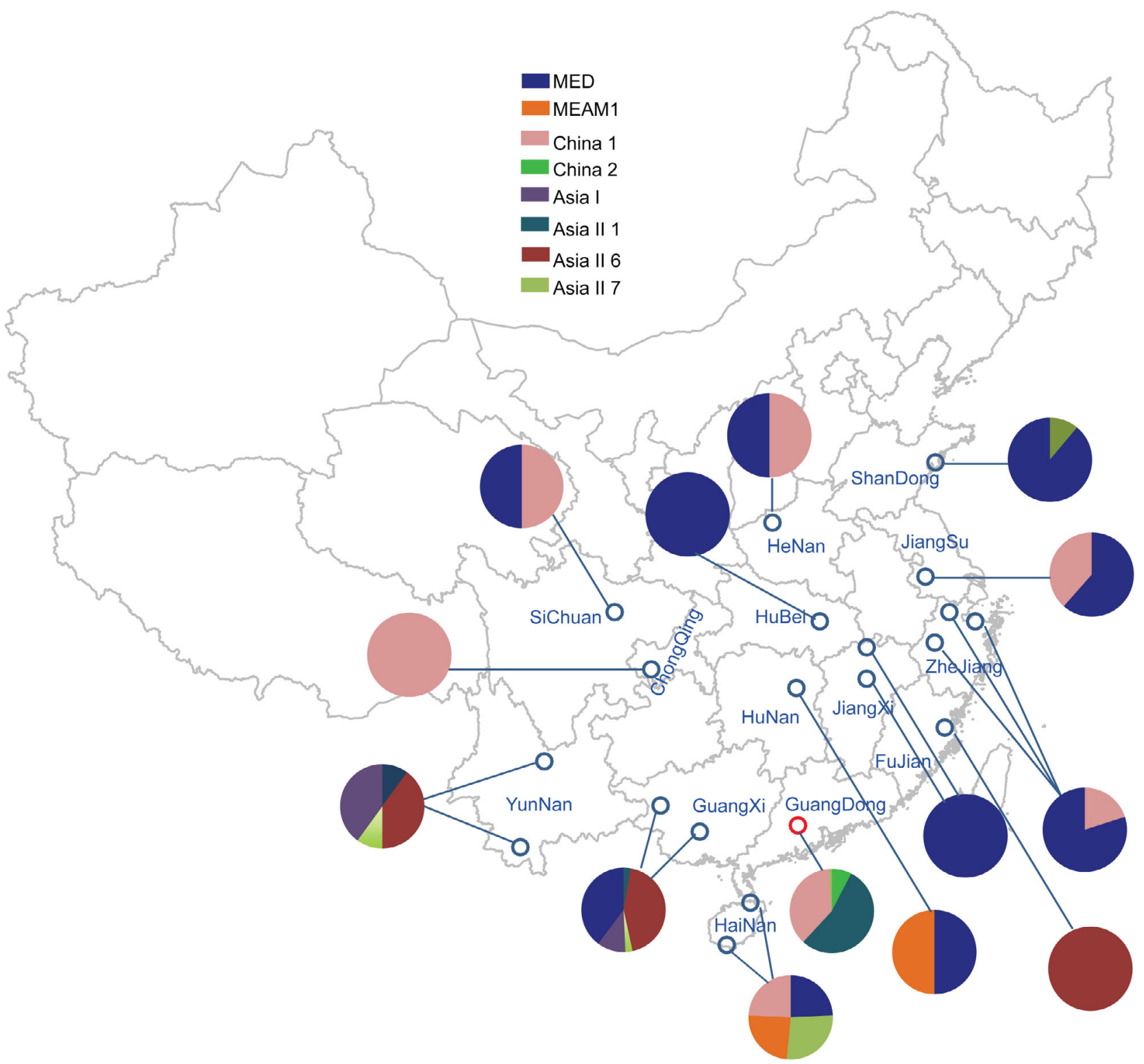

Fig. 2. Distribution of RiTBt in the field. The small red circle indicates the location where RiTBt was found. Each of the pie charts indicates the relative proportion of whitefly species in the $B$. tabaci species complex for each province.

whitefly species, only Asia II 7 and Asia II 1 harboured RiTBt. These two host species are placed in the same clade (Boykin, 2014) based on a recent extensive molecular phylogenetic analysis of the $B$. tabaci cryptic species, suggesting that the new Rickettsia is restricted to a specific species lineage of $B$. tabaci complex.

\section{In vivo localization of RiTBt in whitefly host}

Localizations of RiTBt were examined by fluorescence in situ hybridization (FISH) in oocytes, nymphs, and adults of Asia II 7 B. tabaci. RiTBt was detected inside the bacteriocyte of oocytes, and throughout the whole body of the fourth instar nymphs, as well as females and males of Asia II 7 (Fig. 3.), but was not detected in the negative control of MED individuals (Supporting Information Fig. S1A-D). Notably, RiTBt signals were concentrated in the anterior pole of the oocytes (Fig. 3 . A), indicating that RiTBt can be transmitted to progeny via oocytes. In terms of whitefly organs, RiTBt was visualized in bacteriocytes, midguts, salivary glands, ovaries, and testes (Fig. 4.A-F) of Asia II 7 adults but not in MED (Supporting Information Fig. S1E-I). The presence of RiTBt in the above organs was confirmed by the transmission electron microscope (TEM) (Fig. 4.A5, B4, C4, D5, and E5). 

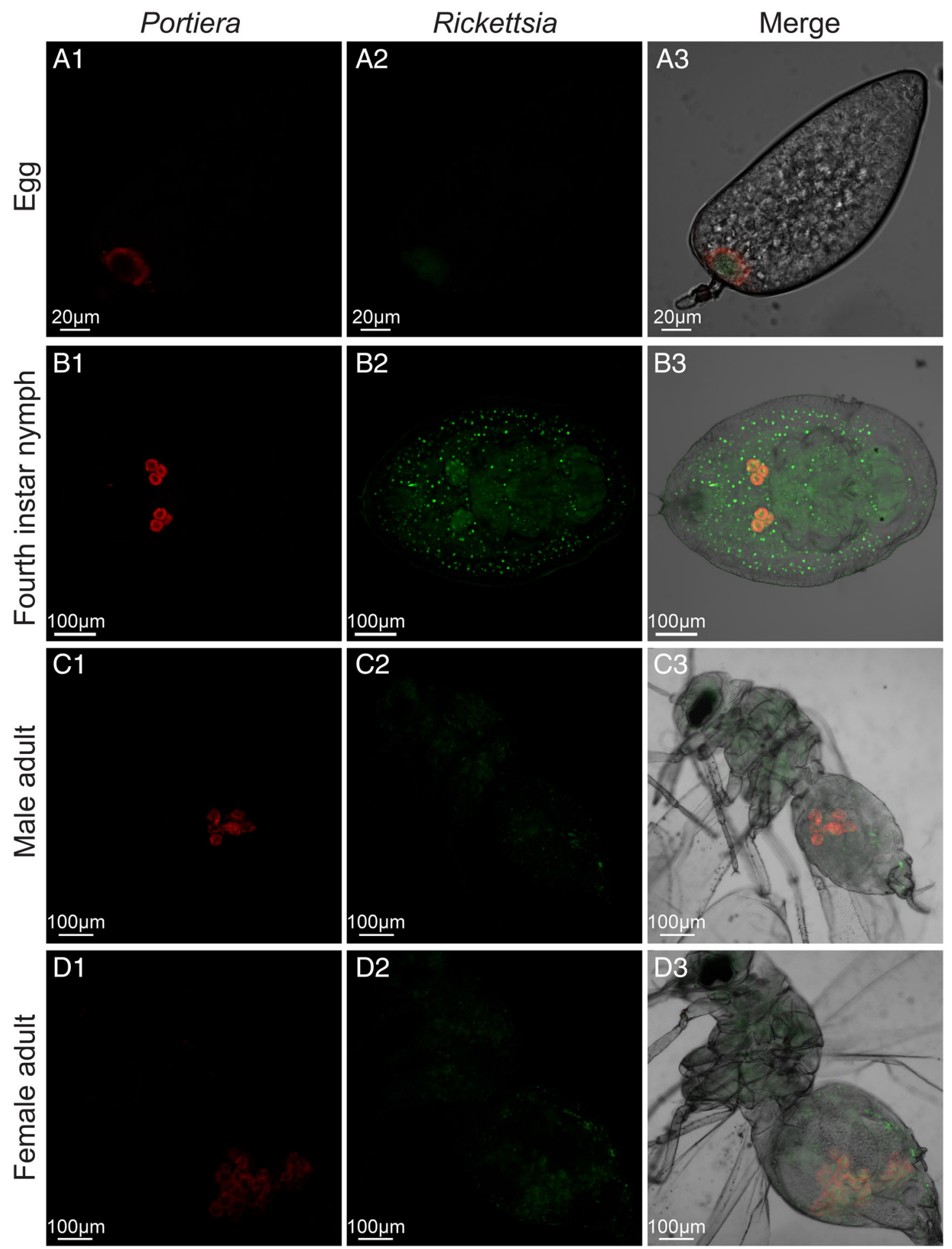

Fig. 3. FISH of RiTBt in B. tabaci Asia II 7. Portiera-specific probe (red) conjugated to cy3, RiTBt-specific probe (green) conjugated to cy5 were used. A1-A3, Egg; B1-B3, Fourth instar nymph; C1-C3, Male adult; D1-D3, Female adult. For the columns, A1, B1, C1, D1, Portiera Channel; A2, B2, C2, D2, 'RiTBt' channel; A3, B3, C3, D3, overlay of Portiera and 'RiTBt' on bright field channels.

\section{Genome and the taxonomy of RiTBt}

The draft genome of RiTBt has a length of 1,485, $811 \mathrm{bp}$ (Table 1), which is slightly larger than that of Torix group member, named RiCNE (Pilgrim et al., 2017), previously sequenced from Culicoides newsteadi (1,456, 172 bp), and Rickettsia_bellii_MEAM1 from the whitefly MEAM1 

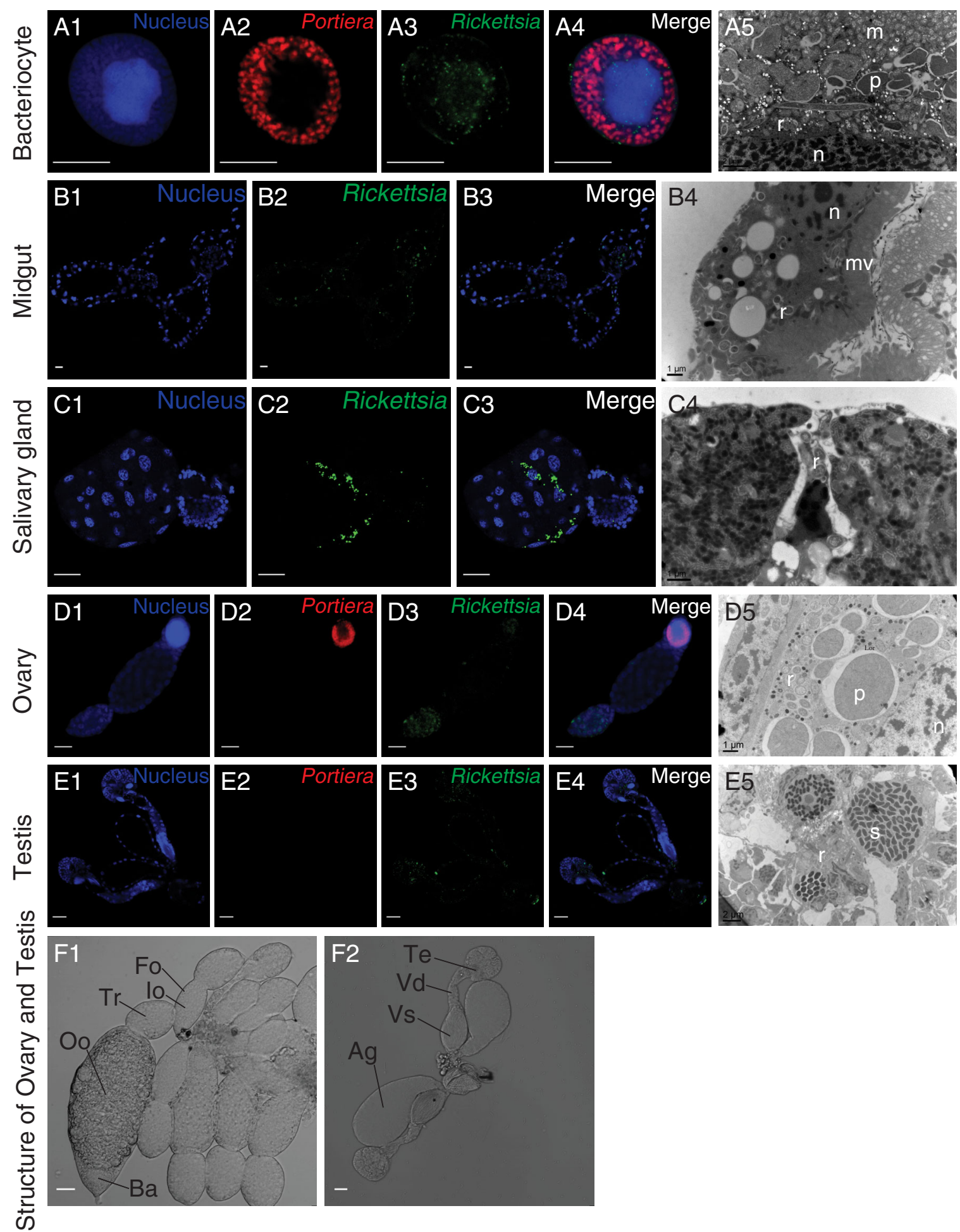

Fig. 4. FISH and TEM show the existence of RiTBt in bacteriocyte, midgut, salivary gland, ovary and testis of Asia II 7. Portiera-specific probe (red) conjugated to cy3 and RiTBt-specific probe (green) conjugated to cy5 were used, and nuclei were stained with DAPI (blue). FISH images: A1-A4, bacteriocyte; B1-B3, midgut; C1-C3, salivary gland; D1-D4, ovary; and E1-E4, testis. F1-F2, germinal system of $B$. tabaci. F1, the structure of ovary; F2, the structure of male reproductive system. Fo, follicular cells; lo, immature oocyte; Tr, trophocytes; Oo, oocyte; Ba, bacteriocyte; Te, testis; Vd, vas deferens; Vs, vesicular siminalis; Ag, accessory glands. TEM images: A5, bacteriocyte; B4, midgut; C4, salivary gland; D5, bacteriocyte in ovary, and E5, testis. For the columns, A1, B1, C1, D1 and E1, 'DAPl' channel; A2, D2 and E2, 'Portiera' channel; A3, B2, C2, D3 and E3, 'RiTBt' channel; A4, B3, C3, D4 and E4, overlay of 'DAPl', 'Portiera aleyrodidarum' and 'RiTBt' on bright field channels. Scale bar $20 \mu \mathrm{m}$. TEM images: A5, bacteriocyte; B4, midgut; C3, salivary gland; D5, bacteriocyte in ovary; E5, testis. In TEM images, $r$ represents Rickettsia; $p$ represents Portiera; $\mathrm{m}$ represents mitochondria; $\mathrm{n}$ represents the nucleus; $\mathrm{mv}$ represents microvilli; s represents sperm. 
Table 1. General statistics and features of RiTBt.

\begin{tabular}{lc}
\hline & E-value $<=1 \mathrm{e}-5$ \\
\hline Total number of scaffolds & 69 \\
Total size (bp) & $1,485,811$ \\
N50 length (bp) & 50,092 \\
Largest contig (bp) & 157,737 \\
Total predicted coding sequences & 1,446 \\
Average length (bp) of coding sequences & 813 \\
Coding density & 0.973 gene per kb \\
GC content (\%) & 33.3 \\
tRNA & 40 \\
rRNA & 3 (5S, 16S, 23S) \\
\hline
\end{tabular}

$(1,378,618 \mathrm{bp})$. The draft genome has a $33.3 \% \mathrm{GC}$ content and comprises 69 scaffolds with an N50 size of approximately 50, 029 bp (Table 1). Benchmarking Universal Single-Copy Orthologues (BUSCO) analyses revealed $90.6 \%$ complete and/or fragmented BUSCOs in the RiTBt genome assembly [In all, there were 130 complete BUSCOs (C; 87.9\%), 124 complete and single-copy BUSCOs (S; 83.8\%), 6 complete and duplicated BUSCOs (D; $4.1 \%), 4$ fragmented BUSCOs (F; 2.7\%), 14 missing BUSCOs (M; 9.4\%), and 148 BUSCO groups.] Totally, 1,446 genes were predicted, in which 1,219 ORFs were annotated by a homology search (Supporting Information Table S2). Of the 1,219 ORFs, 728 genes were assigned to COGs (Supporting Information Table S2 and Fig. S2), five genes were found encoding a tra conjugative DNA transfer element (Supporting Information Table S3) (Ogata et al., 2006), and 14 genes were annotated as coding Vir components (virB4, virB6, virB8-virB11, virD4) of P-like type IV secretion system (P-T4SS), which are highly conserved among Rickettsiales (Supporting Information Table S3). Similar to other Rickettsia genomes, the vir genes were dispersed into three major clusters (scaffold 4: virB4 and virB6; scaffold 10: virB4, virB8-B11, and virD4; scaffold 39: virB4 and virB6) (Gillespie et al., 2009).

In total, 519 core and 459 single-copy protein-coding genes (Supporting Information Fig. S3) were identified among all the 64 complete or draft Rickettsia genomes available (Supporting Information Table S4). Comparison of RiTBt single-copy genes with the 63 other Rickettsia genomes showed that RiTBt has the highest nucleotide similarity to that of the Torix group endosymbiont of RiCNE (on average: $87 \%$ nucleotide identity), but a relatively low nucleotide similarity to those of other Rickettsia species (on average: 66\%-68\% nucleotide identity) (Supporting Information Fig. S4).

To infer a robust phylogenetic relationship of RiTBt relative to other Rickettsiaceae, a Bayesian phylogenomic tree was constructed based on the concatenated alignment of 459 single-copy nuclear genes from the genomes of 64 Rickettsia and its sister genus Orientia
(Supporting Information Table S5). The resulting phylogenomic tree differs from a previous one proposed by Weinert et al. (2009) and provides a global phylogeny of Rickettsia spp. with high bootstrap support for most backbone nodes. RiTBt clustered with RiCNE and was highly divergent from other groups of Rickettsia spp. (Fig. 5.). Moreover, RiTBt and RiCNE are situated at the most ancient lineage in all Rickettsia spp. genomes. Although RiTBt formed a distinct and robust monophyletic Torix clade with RiCNE, its species status with respect to RiCNE is not clear. RiCNE and RiTBt share a $99 \%$ sequence identity for the 16S rRNA gene $(1,461 \mathrm{bp})$, a widely used marker for species delimitation, but this marker does not exhibit sufficiently high resolution for detailed classification (Richter and Rosselló-Móra, 2009). To further classify its species status, average nucleotide identity, which has been widely used to determine species boundaries with cutoffs of $94 \%-96 \%$, was calculated (Konstantinidis and Tiedje, 2005; Richter and Rosselló-Móra, 2009). The average nucleotide identity between RiTBt and RiCNE was $86.25 \%$, notably lower than the $94 \%-96 \%$ criterion for separating species. All the evidence collected here further confirms that RiTBt is a new species under the Torix group.

\section{Gene family expansion or contraction}

Investigation of gene families (orthologous groups) across Rickettsia lineages showed that gene gains were scarce for most Rickettsia lineages, with 0-16 gene family gains at the base of each species-group. In contrast, gene losses were far more common, with 11-46 gene family decays in all groups (Fig. 5.). The most extensive gene family decays occur in the Rickettsia_bellii_MEAM1 (46 gene families lost), whereas RiTBt shows less than half this volume of gene family decays (22 gene families lost) (Fig. 5.). This finding confirmed that gene loss is the leading cause of genome diversification within the Rickettsia genus (Blanc et al., 2007).

\section{Genome synteny and functional profile between 'Torix' and 'Bellii' group species}

We compared genome synteny between the two Torix group species, RiTBt and RiCNE [MWZE00000000], to the two Bellii group species, RML369-c [CP000849] and MEAM1 [CP000849]. The scaffolds of RiTBt displayed little synteny with those of either the Bellii group genomes or RiCNE (Fig. 6.A).

$\mathrm{RiTBt}$ and RiCNE under the Torix group share similar metabolic pathways, which further support the close relationships between the two. In contrast, comparison of RiTBt with Rickettsia_bellii_MEAM1 showed the absence of several crucial pathways in the latter, such as 


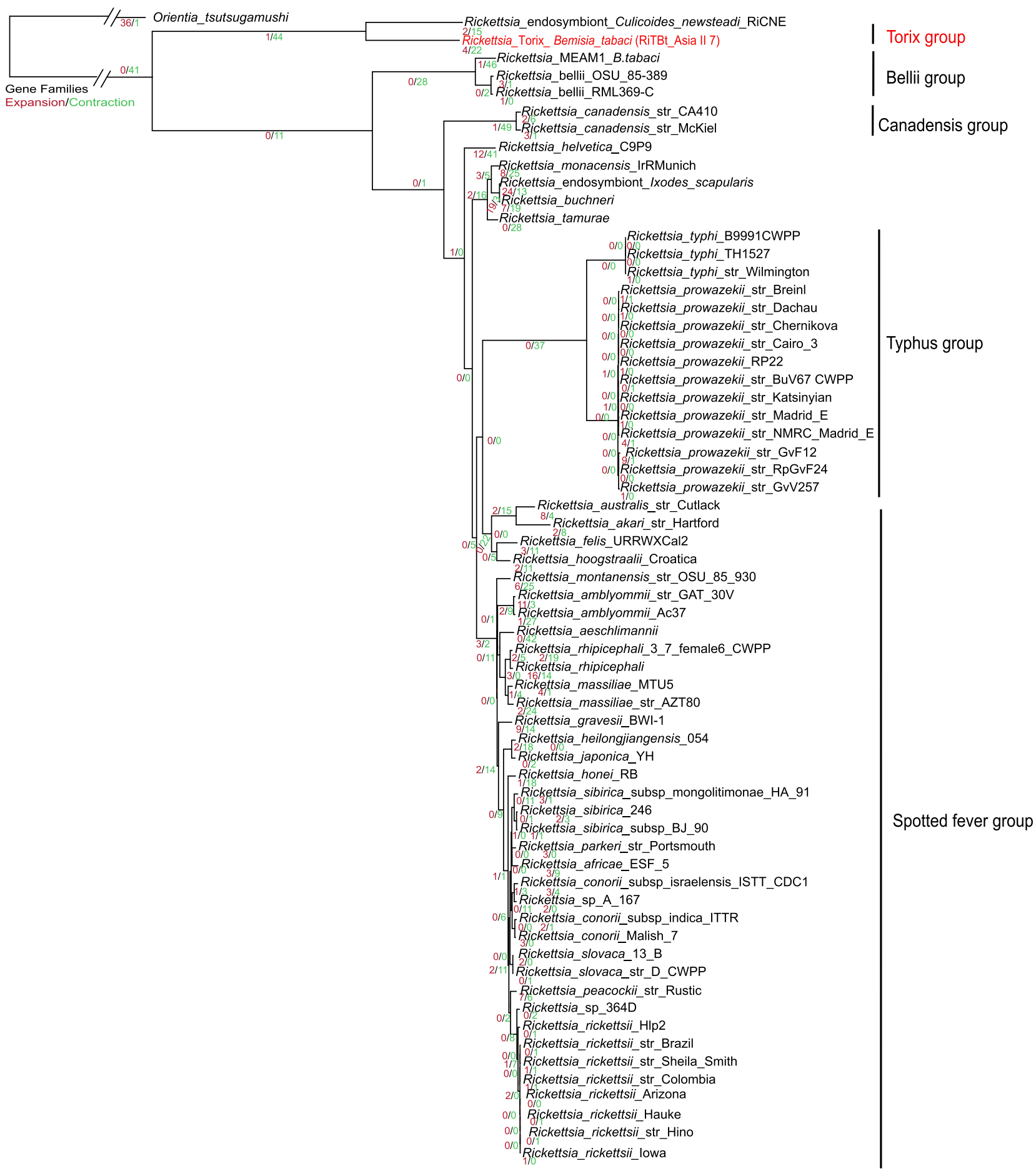

Fig. 5. Gene gain and loss of all known Rickettsia genomes and the phylogenetic position of RiTBt strain (red) based on 459 single-copy nuclear genes. The posterior probabilities are all over 0.85 except for the node $(0.66)$ between the Rickettsia_prowazekii_str_Breinl and the Rickettsia_ prowazekii_str_Dachau.

'Glycolysis (Embden-Meyerhof pathway), glucose', 'Glycolysis, core module involving three-carbon compounds", 'Methionine salvage pathway', 'Pentose phosphate pathway (Pentose phosphate cycle)' and 'Pentose phosphate pathway, non-oxidative phase, fructose 6P' (Fig. 6.B).

\section{Discussion}

Here, we report a bacterium residing in the two indigenous species Asia II 1 and Asia II 7 of the B. tabaci whitefly complex that has not been recorded previously. 
A

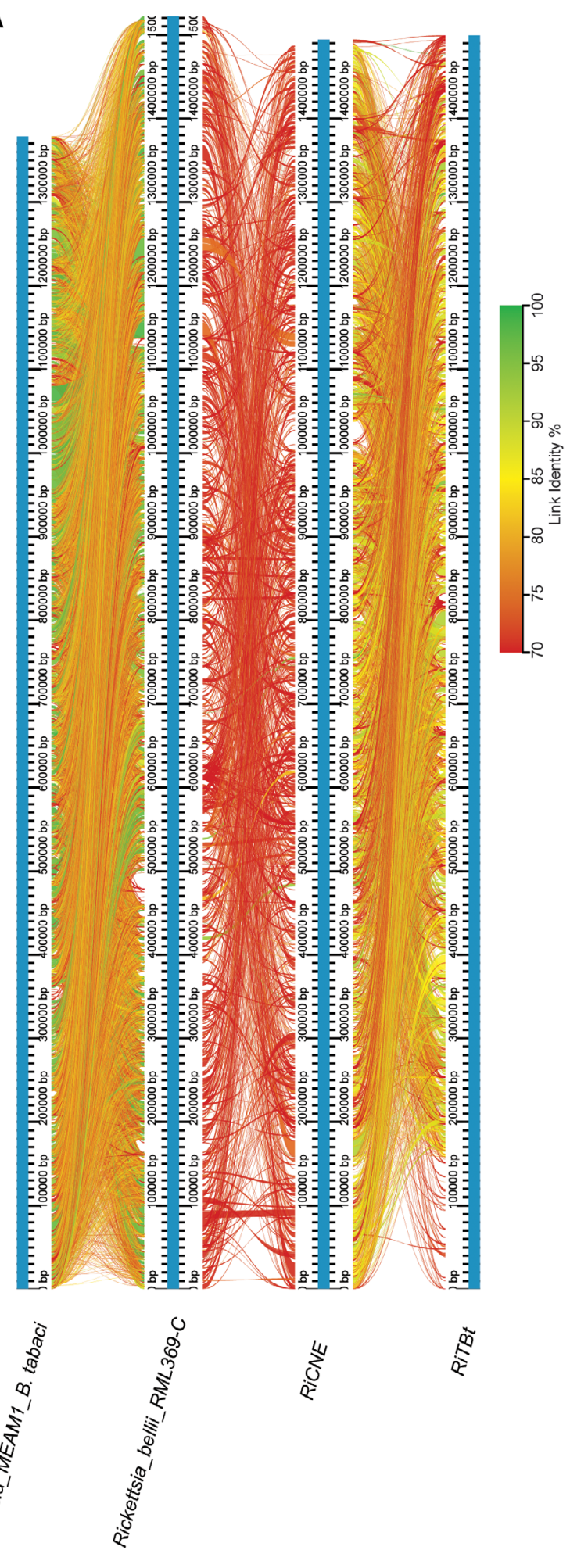

B

\begin{tabular}{l} 
Name \\
3-Hydroxypropionate bi-cycle \\
\hline Adenine ribonucleotide biosynthesis, IMP \\
\hline Ascorbate biosynthesis, animals, glucose-1P \\
\hline Ascorbate biosynthesis, plants, glucose-6P
\end{tabular}

beta-Oxidation

C10-C20 isoprenoid biosynthesis

C1-unit interconversion

C4-dicarboxylic acid cycle, NAD - malic enzyme type

C4-dicarboxylic acid cycle. NADP - malic enzyme type

C5 isoprenoid biosynthesis, mevalonate pathw ay

C5 isoprenoid biosynthesis, non-mevalonate pathw ay

CAM (Crassulacean acid metabolism), dark

CAM (Crassulacean acid metabolism), light

atrate cycle (TCA cycle, Krebs cycle)

Citrate cycle, first carbon oxidation, oxaloacetate

Citrate cycle, second carbon oxidation, 2-oxoglutarate

CMP-KDO biosynthesis

Coenzyme A biosynthesis, pantothenate

Cysteine biosynthesis, methionine

D-galactonate degradation, De Ley-Doudoroff pathw ay, D-galactonate

Dicarboxylate-hydroxybutyrate cycle

dTDP-L-rhamose biosynthesis

Ectoine biosynthesis, aspartate

Ethylene biosynthesis, methionine

Ethylmalonyl pathw ay

Fatty acid biosynthesis, elongation

Fatty acid biosynthesis, initiation

Formaldehyde assimilation, serine pathwey

GABA (gamma-Aminobutyrate) shunt

Galactose degradation, Leloir pathw ay, galactos

Gluconeogenesis, oxaloacetate

Glucuronate pathw ay (uronate pathway)

Glycolysis (Enbden-Meyerhof pathw ay), glucose

Glycolysis, core module involving three-carbon compounds

Glyoxylate cycle

Guanine ribonucleotide biosynthesis IMP

Heme biosynthesis, glutamate

Hydroxypropionate-hydroxybutylate cycle

Inosine monophos phate biosynthesis, PRPP + glutamin

Inositol phosphate metabolism, $\mathbf{n s}(1,3,4,5) \mathrm{P4}$

Isoleucine biosynthesis, threonine

Ketone body biosynthesis, acetyl-CoA

Leucine degradation, leucine

Lipopolysaccharide biosynthesis, inner core

Lipopolysaccharide biosynthesis, KDO2-lipid

Lysine biosynthesis, acetyl-DAP pathw ay, aspartate

Lysine biosynthesis, DAP aminotransferase pathw ay, aspartate

Lysine biosynthesis, DAP dehydrogenase pathw ay aspartate

Lysine biosynthesis, succinyl-DAP pathw ay, aspartate

Lysine degradation, lysine

Menaquinone biosynthesis, chorismate

Methionine biosynthesis, apartate

Methionine degradation

Methionine salvage pathw

Methylaspartate cycle

Ornithine biosynthesis, glutamate

Pantothenate biosynthesis, valine/L-aspartate

Pentose phos phate pathw ay (Pentose phosphate cycle)

Pentose phosphate pathw ay non-oxidative phase, fructose $6 P$

Phos phatidylethanolarnine (PE) biosynthesis, PA

Photorespiration

Pmeloyl-ACP biosynthesis, BioC-BioH pathw ay, malonyl-ACP

Propanoyl-COA metabolism, propanoyl-COA

PRPP biosynthesis, ribose 5P

Pyrimidine deoxyribonuleotide biosynthesis, CDPICTP

Pyrimidine ribonucleotide biosynthesis. UMP

Pyruvate oxidation, pyruvate

Reductive acetyl-CoA pathw ay (Wood-Ljungdahl pathw ay)

Reductive citrate cycle (Arnon-Buchanan cycle)

Reductive pentose phosphate cycle (Calvin cycle)

Reductive pentose phosphate cycle, glyceraldehyde-3P

Reductive pentose phosphate cycle, ribulose-5P

Semi-phosphorylative Entner-Doudoroff pathw ay, gluconate

Telratydroflate biosynhesis, GIP

Threonine blosynthesis, aspartate

Triacylglycerol biosynthesis

Ubiquinone biosynthesis, eukaryotes, 4-hydroxybenzoate

Ubiquinone biosynthesis, prokaryotes, chorismate
Valine/isoleucine biosynthesis, pyruvate

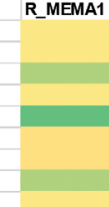

RiGNE

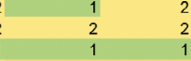

$\begin{array}{lll}1 & 6 \\ 2 & 6\end{array}$

$\begin{array}{ll}1 & 0 \\ 4 & 4\end{array}$

$\begin{array}{ll}2 & 1 \\ 1 & 1 \\ 1 & 1\end{array}$

$\begin{array}{ll}2 & 2 \\ 7 & 7\end{array}$

$\begin{array}{ll}2 & 2 \\ 1 & 1\end{array}$

43

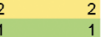

1

$2 \quad 2$

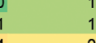

2

$3 \quad 2$

$\begin{array}{ll}2 & 2 \\ 6 & 6\end{array}$

$\begin{array}{ll}6 & 6 \\ 1 & 0\end{array}$

$\begin{array}{ll}1 & 9 \\ 2 & 2\end{array}$

2

1

4

$\begin{array}{ll}5 & 4 \\ 4 & 4\end{array}$

16
-1

2

Fig. 6. Genome synteny and functional profile between 'Torix' and 'Bellii' group species. A. Alignment of RiTBt, Rickettsia_MEAM1_B. tabaci, RiCNE and Rickettsia bellii genomes. B. Assessment of the metabolic potential of RiTBt genome and comparison to the Rickettsia bellii MEAM1 and RiCNE. R_MEAM1 denotes Rickettsia_bellii_MEAM1. The names of the pathways that differ between RiTBt and the other two Rickettsia spp. are written in red, and different colours of the cells in the table indicate the varying number of genes being classified for each of the pathways: green indicates fewer and red indicates more genes. 
Phylogenomic analysis based on hundreds of single-copy nuclear genes showed that this bacterium belongs to Rickettsiae with a close relationship with species of the Torix group. Through field survey, in vivo localization, and genomic comparative analysis, we named this newly found endosymbiotic bacterium in whiteflies as 'Candidatus Rickettsia_Torix_Bemisia_tabaci (RiTBt)'.

\section{The range of hosts harbouring Rickettsia of the Torix group}

Apart from whiteflies, Torix group Rickettsia have been reported previously in an array of phylogenetically distant invertebrate vectors including the rice leafhopper Nephotettix cincticeps (AB702995) (Noda et al., 2012), true bug Macrolophus sp. (HE583203) (Machtelinckx et al., 2012), water beetles Deronectes aubei (FM955310), Deronectes semirufus (FM955311), and Deronectes delarouzei (FM955312) (Küchler et al., 2009), leeches Hemiclepsis marginata (AB066352), Torix tukubana (AB113214), and Torix tagoi (AB066351) (Kikuchi et al., 2002; Kikuchi and Fukatsu, 2005), cicada Platypleura kaempferi (KR911839), sand fly Phlebotomus chinensis) (KX363668), mite Lutzomyia apache (EU223247) (Reeves et al., 2008), crane fly Limonia chorea (AF322442) (Perotti et al., 2006), ice worm Mesenchytraeus solifugus glacier (AB991365.1), and booklice Cerobasis guestfalica (DQ652595)] (Supporting Information Fig. S5). Interestingly, many of these vectors are aquatic invertebrates whose abundance has long been known to be closely tied to water quality, such as midges, water beetles, ice worms, crane flies, leeches, and sandflies. However, the remaining vectors, such as rice leafhoppers, cicadas, true bugs, and whiteflies, are sap-sucking insects. The idea that horizontal transmission occurs in nature between distant relatives is widely accepted (Caspi-Fluger et al., 2012; Russell et al., 2003). Thus, whiteflies might have captured this Rickettsia via plant sap, which contains this Rickettsia excreted by insects of the same order, such as rice leafhoppers, cicadas, or true bugs. More investigations are warranted to explore its possible transmission routes among ecologically and phylogenetically disparate hosts.

\section{Implications of the localization of RiTBt in B. tabaci}

FISH and TEM consistently showed the presence of an intracellular Rickettsia-like structure located in the whole-body cavity, as well as midgut, salivary gland, bacteriocyte, ovary, and testis of Asia II 7. The distribution throughout the host body implies that this Rickettsia has evolved to elude immune defences and survive in the body tissues of $B$. tabaci whiteflies without harming the host. The fact that the RiTBt can be detected inside whitefly eggs, nymphs, and adults indicates vertical transmission.
A higher density of RiTBt was detected in the midgut, especially in the filter chamber of whitefly midgut than that of other organs/tissues, a pattern of distribution similar to that reported for Rickettsia_bellii_MEAM1 (Kliot et al., 2014). We also observed high densities of RiTBt in the salivary gland - as phloem-feeding insects whiteflies of the B. tabaci complex are potentially able to overwhelm plant defence mechanisms using their saliva (Will et al., 2013; $\mathrm{Xu}$ et al., 2019). Whether symbionts in the salivary glands of whitefly are involved in host defence to plant resistance warrants exploration.

Functions of Rickettsia in the biology of several phloem-feeding insects have been reported, including tolerance to heat stress, promotion of reproduction and spread, and resistance to insecticides (Ferrari et al., 2004; Ghanim and Kontsedalov, 2009; Brumin et al., 2011; Himler et al., 2011). The effect of RiTBt on the biology of whiteflies in the $B$. tabaci species complex is yet to be investigated, but the observations of its spatial and subcellular localization provided here may serve as clues for future effort on this topic.

\section{Stable taxonomy based on genome data}

Phylogenomic estimation is central to understand biodiversity, evolution, and ecology and provides a higher taxonomic resolution than phylogenies obtained from single marker genes (Cao and Sun, 2016). To accurately describe and accommodate RiTBt, we compared previously defined Rickettsia species with RiTBt to conduct a global phylogenomic analysis. RiTBt was recognized as a new, independent species under the Torix group based on both phylogenomic estimation and the recently proposed delimitation criteria of bacterial species (Konstantinidis and Tiedje, 2005; Richter and RossellóMóra, 2009). The results not only confirmed the evolutionary relationship of RiTBt with other species in the Rickettsia genus but also filled a major phylogenetic gap of species diversity in the Torix clade.

\section{Implication of comparison between gene families and metabolic pathways}

Differences in the size of gene families between organisms have generally been attributed to their adaptive evolution (Hahn et al., 2005). In a global view of Rickettsia genome family evolution, our data indicate that considerable contraction has occurred in the gene families of RiTBt, illustrating a large amount of genetic downsizing relative to Orientia tsutsugamushi, an obligate intracellular bacterium that also exhibits genome reduction relative to free-living bacteria (Cole et al., 2001). The most extensive genome degradation (37 gene family decays) reported previously was from $R$. prowazekii (Anderssen 
et al., 1998). Our analysis illustrates that much higher genome degradation has occurred in Rickettsia genome families, as shown by the 46 gene family decays of Rickettsia_bellii_MEAM1.

Similar to gene families, certain essential metabolic pathways, such as 'Glycolysis (Embden-Meyerhof pathway), glucose', 'Glycolysis, core module involving threecarbon compounds', are absent in Rickettsia_bellii_MEAM1 compared to RiTBt. Both species of the Torix group retain a crucial pentose phosphate pathway (Pilgrim et al., 2017), which is associated with maintenance of carbon homoeostasis, provision of precursors for nucleotide and amino acid biosynthesis, reduction of molecules for anabolism, and decline of oxidative stress (Stincone et al., 2015). Similar to an uncultured pathogenic endosymbiont 'Candidatus Liberibacter asiaticus' in psyllid (Jain et al., 2017), glycolysis and the pentose phosphate pathways are both absent in Rickettsia_bellii_MEAM1, indicating that this Rickettsia could be an energy scavenger and thus import ATP from its host (Jain et al., 2017). The absence of glycolysis and the pentose phosphate pathways in Rickettsia_bellii_MEAM1 could be complemented by the host (Driscoll et al., 2017) or other microbial symbionts in the host. The above analyses suggest that RiTBt is more recently acquired than Rickettsia_bellii_MEAM1 by its host and is thus less co-evolved but still shows signs of adaptation to endosymbiosis in its host strain distribution and gene family loss in comparison to the Orientia outgroup.

\section{RiTBt, a new species of Rickettsia associated with}

B. tabaci

Rickettsia_bellii_MEAM1 is the first Rickettsia species described residing in $B$. tabaci whiteflies (Gottlieb et al., 2006; Singh et al., 2012). Comparison of characteristics of RiTBt with those of Rickettsia_bellii_MEAM1, presumably the closest relative to RiTBt, based on their being associated with the same insect host (Caspi-Fluger et al., 2011; Chen et al., 2016) shows the following differences between the two:

(i) Genome features and phylogenic classification. The genomes' differences in size, number of transposes, gene arrangement, metabolic pathways, gene families, and phylogenetic position between RiTBt and Rickettsia_bellii_MEAM1 are sufficient to classify RiTBt as a new species. RiTBt is more closely related to a Rickettsia from another host species than to the other Rickettsia described in whiteflies, and its genome size and content suggest that it is a more recent acquisition than Rickettsia_bellii_MEAM1.

(ii) Spatial and subcellular localization in host body. RiTBt resides in the midgut, bacteriocyte, ovary, male reproductive system, as well as in nucleus and muscle tissues of its host based on our observations, while Rickettsia_bellii_MEAM1 has two patterns of distribution - a 'scattered' pattern where the Rickettsia is localized throughout the whitefly hemocoel excluding bacteriome, and a 'confined' pattern where the Rickettsia is restricted to the bacteriocytes, both of which differ from that of RiTBt.

(iii) Host range. RiTBt has been found so far only in indigenous species of the $B$. tabaci complex, Asia II 1 and Asia II 7, while Rickettsia_bellii_MEAM1 has been found in both indigenous and invasive species MEAM1, Asia II 3, and China 1. Notably, their hosts do not overlap suggesting specific mechanisms of each for adaptation to its hosts, suggesting specific mechanisms of adaptation for different whitefly host species.

The evidence obtained from the comparison shows that the newly detected Rickettsia is a new species of the bacterium residing in whiteflies of the $B$. tabaci species complex. The differences in gene family and metabolic pathways between 'Candidatus Rickettsia_Torix_ Bemisia_tabaci (RiTBt)' and Rickettsia_bellii_MEAM1, in particular, the absence of some vital gene families in Rickettsia_bellii_MEAM1, indicate that the two species of Rickettsia may differ in their symbiosis with their hosts. Detailed analyses of these differences may reveal distinct roles of the two species of Rickettsia in the biology of the host whiteflies.

\section{Materials and methods}

Insect materials

A colony of B. tabaci Asia II 7 (identified from its mitochondrial cytochrome oxidase 1 [ $\mathrm{mtCO} /$ gene sequence; GenBank accession no. KM821541) was initially collected from the host plant Hibiscus rosa-sinensis (L.) in Guangzhou, Guangdong province of China, in September 2013. It was maintained on cotton [Gossypium hirsutum (Malvaceae) cv. Zhe-Mian 1793] under the conditions of $26 \pm 1{ }^{\circ} \mathrm{C}, 60 \% \pm 10 \%$ relative humidity and a photoperiod of $14 \mathrm{~h}$ light and $10 \mathrm{~h}$ darkness (Liu et al., 2007), for subsequent genome sequencing, FISH and TEM observations.

To identify the presence of the RiTBt in field whitefly populations, 1-46 adult whiteflies were taken from each of 57 B. tabaci samples that were collected from 22 localities of 14 provinces of China, from June to September 2015 (Fig. 2. and Supporting Information Table S1). In total, 512 adult whiteflies were screened. These wild whitefly specimens were initially stored in $95 \%$ ethanol and subsequently kept at $-80^{\circ} \mathrm{C}$ until DNA extraction. 
DNA extraction, PCR amplification, and sequencing

Individual adult DNA extraction and the cryptic species diagnosis were done using the method of Bing et al. (2013). The phylogenetic relationships of the RiTBt with other symbionts were estimated with three widely used markers: a $1.4 \mathrm{~Kb}$ fragment of the $16 S \mathrm{SRNA}$ gene with primers 16S 27F (5'-CTACGGCTACCTTGTTACGA-3') and 16S 1494R (5'-ATTGCTTCCCTCTGTGGAT-3') (Weisburg et al., 1991), a $0.5 \mathrm{~Kb}$ fragment of the gltA gene with primers gltA $\mathrm{F}$ (5'-ACATGCAGACCATGAGCAGA-3') and gltA $\mathrm{R}$ (5'-CATTTCATTCCATTGTGCCATC-3') (Li et al., 2011), and a $1.6 \mathrm{~Kb}$ fragment of the groEL gene with newly designed primers groEL $F$ (5'-CACCDA AAATTACTAAAGATGG-3') and groEL $\mathrm{R}$ (5'-TAGAART CCATWCCKCCCATWC- ${ }^{\prime}$ ).

To detect the presence of the RiTBt in whitefly populations, specific 16S rRNA primers, 16S $\mathrm{F}$ (5'-GATTAATTTAGGGCTTGCTCTG-3') and 16S R (5'-ATTGCTTCCCTCTGTGGAT-3'), were designed by comparing a wide range of Rickettsia spp., and for the further confirmation, the relative amplicons were sent for sequence. 16S rRNA sequences were downloaded from GenBank. The size of the PCR product is $1.1 \mathrm{~Kb}$. Cycling condition was $94^{\circ} \mathrm{C}$ for $3 \mathrm{~min}, 34$ cycles of $94^{\circ} \mathrm{C}$ for $30 \mathrm{~s}$, $50-53^{\circ} \mathrm{C}$ for $30 \mathrm{~s}, 72^{\circ} \mathrm{C}$ for $2 \mathrm{~min}$, and a final cycle of $72^{\circ} \mathrm{C}$ for $10 \mathrm{~min}$. The amplicons were purified using a QIAGEN PCR purification kit (Germany) and submitted for Sanger sequencing in both directions.

\section{Fluorescence in situ hybridization}

Eggs, fourth instar nymphs, and adults of $B$. tabaci were subjected to FISH following the approach of Gottlieb et al. (2006) and Bing et al. (2013). As for dissected organs, FISH was conducted as previously described by Ghanim et al. (2009) with a slight modification. The obligate endosymbiont 'Ca. Portiera aleyrodidarum' (Portiera) occurs exclusively inside bacteriocytes throughout the host life cycle of all B. tabaci species (Gottlieb et al., 2006; Bing et al., 2013); therefore, it provides a good control for FISH. Thus, the widely used probe BTP1-Су3 (5'-Су3-TGTCA GTGTCAGCCCAGAAG-3') was chosen to target the $16 S$ rRNA of the Portiera (Gottlieb et al., 2006), while a new probe, R-Cy5 (5'-Cy5-TCCACGTCACCGTATTGC$\left.3^{\prime}\right)$, was developed for detection of Ricketssia strains using Primer3 software (http://bioinfo.ut.ee/primer3-0.4.0/ primer3/) based on the 16S rRNA sequence from Rickettsia-like strains. The specificity of detection was confirmed using Rickettsia-free whitefly $B$. tabaci MED as negative controls. Fluorescence was visualized on ZEISS LSM780 at Bio-ultrastructure Analysis Laboratory of the Analysis Center of Agrobiology and Environmental Sciences, Zhejiang University.

\section{Transmission electron microscopy}

To observe the localization of RiTBt in cells or tissues, TEM was conducted on midguts, salivary glands, bacteriocytes, ovaries, and testes, dissected from adult specimens of Asia II 7. TEM analysis was performed as follows: (i) Double fixation: samples were firstly fixed with $2.5 \%$ glutaraldehyde in PBS buffer for $4 \mathrm{~h}$, then rinsed three times in PBS buffer for 15 min each, and postfixed with osmium tetroxide in PBS buffer for $15 \mathrm{~min}$. (ii) Dehydration: the samples were dehydrated in an ascending ethanol series for $20 \mathrm{~min}$ before transferring to absolute acetone. (iii) Infiltration: the samples were placed in 1:1 mixture of absolute acetone and Spurr resin overnight. (iv) Embedding and ultrathin sectioning: the samples were placed in an Eppendorf tube containing pure Spurr resin and incubated at $70^{\circ} \mathrm{C}$ for $9 \mathrm{~h}$. In the end, samples were sectioned in a LEICA EM UC7 ultramicrotome, and sections were stained with aqueous uranyl acetate and lead citrate for $5 \mathrm{~min}$ and $10 \mathrm{~min}$ respectively. These sections were observed under a Hitachi Model H-7650 TEM at Bio-ultrastructure Analysis Laboratory of the Analysis Center of Agrobiology and Environmental Sciences, Zhejiang University.

\section{Isolation, amplification, and sequencing of} endosymbiont genome

The dissection of bacteriomes, genomic DNA isolation, library preparation, and sequencing was conducted as described in the study by Rao et al. (2015). Briefly, nymphs with obvious green-yellow coloured bacteriomes were collected and dissected in PBS $\left(\mathrm{GIBCO}^{\circledR}\right.$, Invitrogen, USA) using autoclaved tools. PBS containing endosymbiont cells were then divided into six separate samples and amplified by multiple displacement amplification separately using the REPLI-g UltraFast Mini Kit (Qiagen, Germany) with slight modifications. Subsequently, the yield of the six copies was mixed with equal concentrations to construct one paired-end library $(2 \times 150$ bp paired reads) with an average insert size of 400-500 bp. The sample was then sequenced on Illumina HiSeq2000 (Biozeron Biotech, Shanghai), with 8 million reads generated.

\section{Genome assembly}

Raw FASTQ data were generated by the lllumina basecalling software CASAVA v1.8.2 (http://supportillumina. $\mathrm{com} /$ sequencing/sequencing_software/casava.ilmn). Quality assessment and filtering of the Illumina reads were performed using FASTQC v0.10.1 (Andrews, 2010) and FASTX toolkit v0.0.13 (Gordon, 2011). Contaminating reads, containing adaptor sequences or primer 
sequences were identified by SeqPrep (https://github. com/jstjohn/SeqPrep) with the parameters: '-q 20-L 75-B AGATCGGAAGAGCGTCGTGT-A

AGATCGGAAGAGCACACGTC'. Sickle (https://github. com/najoshi/sickle) was applied for reads trimming with default parameters. Through the above quality control processes, clean reads were obtained for a preliminary assembly by SPAdes version 3.10 (Bankevich et al., 2012) with multiple k-mer sizes under 'careful' mode. The parameters for filtering redundant reads/contigs followed the approach described by Pilgrim et al. (2017). The final assembly was subjected to a final decontamination step to remove the contigs that had high similarity with the other endosymbionts found in $B$. tabaci. The remaining scaffolds were used for downstream analysis.

The assessment of completeness of the genome assembly, prediction of coding sequences and tRNAs identification and annotations were conducted using the method used by Pilgrim et al. (2017).

\section{Orthologue identification, molecular phylogeny and evolutionary analyses of gene family}

We downloaded 64 Rickettsia genomes and one outgroup Orientia tsutsugamushi genome from PATRIC (Wattam et al., 2014) for conducting the comparative genomic analysis. OrthoMCL (Li et al., 2003) was used to generate orthologous groups (OGs) of protein families from the 64 complete or nearly complete genomes. From this analysis, proteins were designated unique, core, or non-conserved based on their distribution across Rickettsia genomes. A set of 459 single-copy nuclear genes were selected to concatenate and were aligned in MUSCLE with default parameters (Edgar, 2004). Gblocks (Castresana, 2002) was used to mask regions of poor alignment (heterogeneous length regions). All modified alignments were then concatenated.

Phylogenetic trees for either single genes or largescale concatenated sequences were constructed using the program MrBayes ver. 3.2 (Ronquist et al., 2012). PartitionFinder2 (Lanfear et al., 2016) was used to choose best-fit substitution models for each nucleotide and protein sequence alignment. Computational Analysis of Gene Family Evolution (CAFE) (De Bie et al., 2006), a tool using a stochastic birth and death process to model the evolution of gene family size over a phylogeny, was employed to study gene family gain and loss.

\section{Comparative genomic analysis}

Genome rearrangement was assessed using AliTV (Ankenbrand et al., 2017). Metabolic potential of the RiTBt was evaluated using the Metabolic and Physiological Evaluator (MAPLE-2.1.0) (Takami et al., 2016).

\section{Acknowledgements}

The authors thank Professor Myron Zalucki for critically reading an earlier version of the manuscript. The authors also thank San-Ling Wu, Zhejiang University, and Liang Zeng, Shanghai Biozeron Biotechnology Co. Ltd, for providing sequencing platform and bioinformatics support. This work was supported by Bill \& Melinda Gates Foundation (African cassava whitefly project, OPP1058938), China Postdoctoral Science Special Foundation (Project no. 517000-X91609), and Chinese Postdoctoral Science Foundation (Project no. 517000-X91502).

\section{Data accessibility}

The sequences of the RiTBt bacteria genes obtained in this study are deposited in GenBank under the accession numbers MG063879 (16S rRNA), MG063880 ( $g$ ltA) and MG063881 (groEL). The whole-genome shotgun project has been deposited at GenBank under the accession number NVXZ00000000.

\section{References}

Anderssen, S., Zomorodipour, A., and Andersson, J. (1998) The complete genome sequence of the obligate intracellular parasite Rickettsia prowazekii. Nature 396: 133-140.

Andrews, S. (2010) FastQC: a quality control tool for high throughput sequence data. URL http://www.bioinformatics. babraham.ac.uk/projects/fastqc/'?

Ankenbrand, M.J., Hohlfeld, S., Hackl, T., and Förster, F. (2017) AliTV-interactive visualization of whole genome comparisons. PeerJ Computer Science 3: e116.

Bankevich, A., Nurk, S., Antipov, D., Gurevich, A.A., Dvorkin, M., Kulikov, A.S., et al. (2012) SPAdes: a new genome assembly algorithm and its applications to singlecell sequencing. J Comput Biol 19: 455-477.

Baumann, P. (2005) Biology of bacteriocyte-associated endosymbionts of plant sap-sucking insects. Annu Rev Microbiol 59: 155-189.

Bing, X.L., Yang, J., Zchori-Fein, E., Wang, X.W., and Liu, S. S. (2013) Characterization of a newly discovered symbiont of the whitefly Bemisia tabaci (Hemiptera: Aleyrodidae). Appl Environ Microbiol 79: 569-575.

Blanc, G., Ogata, H., Robert, C., Audic, S., Suhre, K., Vestris, G., et al. (2007) Reductive genome evolution from the mother of Rickettsia. PLoS Genet 3: e14.

Boykin, L.M. (2014) Bemisia tabaci nomenclature: lessons learned. Pest Manag Sci 70: 1454-1459.

Brumin, M., Kontsedalov, S., and Ghanim, M. (2011) Rickettsia influences thermotolerance in the whitefly Bemisia tabaci B biotype. Insect Sci 18: 57-66.

Cao, C.C., and Sun, X. (2016) Combinatorial pooled sequencing: experiment design and decoding. Quant Biol 4: $36-46$.

Caspi-Fluger, A., Inbar, M., Mozes-Daube, N., Mouton, L., Hunter, M.S., and Zchori-Fein, E. (2011) Rickettsia 'in' and 'out': two different localization patterns of a bacterial symbiont in the same insect species. PLoS One 6: e21096. 
Caspi-Fluger, A., Inbar, M., Mozes-Daube, N., Katzir, N., Portnoy, V., Belausov, E., et al. (2012) Horizontal transmission of the insect symbiont Rickettsia is plant-mediated. Proc $R$ Soc $B$ 279: 1791-1796.

Castresana, J. (2002) Gblocks, v. 0.91 b. Online version.

Chen, W., Hasegawa, D.K., Kaur, N., Kliot, A., Pinheiro, P. V., Luan, J., et al. (2016) The draft genome of whitefly Bemisia tabaci MEAM1, a global crop pest, provides novel insights into virus transmission, host adaptation, and insecticide resistance. BMC Biol 14: 110.

Cole, S., Eiglmeier, K., Parkhill, J., James, K., Thomson, N., Wheeler, P., et al. (2001) Massive gene decay in the leprosy bacillus. Nature 409: 1007-1011.

De Bie, T., Cristianini, N., Demuth, J.P., and Hahn, M.W. (2006) CAFE: a computational tool for the study of gene family evolution. Bioinformatics 22: 1269-1271.

Douglas, A.E. (2006) Phloem-sap feeding by animals: problems and solutions. J Exp Bot 57: 747-754.

Driscoll, T.P., Verhoeve, V.I., Guillotte, M.L., Lehman, S.S., Rennoll, S.A., Beier-Sexton, M., et al. (2017) Wholly Rickettsia! Reconstructed metabolic profile of the quintessential bacterial parasite of eukaryotic cells. MBio 8: e00859-e00817.

Edgar, R.C. (2004) MUSCLE: multiple sequence alignment with high accuracy and high throughput. Nucleic Acids Res 32: 1792-1797.

Everett, K.D.E., Thao, M., Horn, M., Dyszynski, G.E., and Baumann, P. (2005) Novel chlamydiae in whiteflies and scale insects: endosymbionts 'Candidatus Fritschea bemisiae' strain Falk and 'Candidatus Fritschea eriococci' strain elm. Int J Syst Evol Microbiol 55: 1581-1587.

Ferrari, J., Darby, A.C., Daniell, T.J., Godfray, H.C.J., and Douglas, A.E. (2004) Linking the bacterial community in pea aphids with host-plant use and natural enemy resistance. Ecol Entomol 29: 60-65.

Ghanim, M. (2016) Investigating the role of Rickettsia in tomato yellow leaf curl virus interactions with its whitefly vector Bemisia tabaci. In Proceedings of the Plant and Animal Genome XXIV Conference.

Ghanim, M., Brumin, M., and Popovski, S. (2009) A simple, rapid and inexpensive method for localization of tomato yellow leaf curl virus and potato leafroll virus in plant and insect vectors. J Virol Methods 159: 311-314.

Ghanim, M., and Kontsedalov, S. (2009) Susceptibility to insecticides in the $Q$ biotype of Bemisia tabaci is correlated with bacterial symbiont densities. Pest Manag Sci 65: 939-942.

Gillespie, J.J., Ammerman, N.C., Dreher-Lesnick, S.M., Rahman, M.S., Worley, M.J., Setubal, J.C., et al. (2009) An anomalous type IV secretion system in Rickettsia is evolutionarily conserved. PLoS One 4: e4833.

Gillespie, J.J., Williams, K., Shukla, M., Snyder, E.E., Nordberg, E.K., Ceraul, S.M., et al. (2008) Rickettsia phylogenomics: unwinding the intricacies of obligate intracellular life. PLoS One 3: e2018.

Gordon, A. (2011) FASTX-Toolkit. Computer program distributed by the author. http://hannonlab.cshl.edu/fastx_toolkit/.

Gottlieb, Y., Ghanim, M., Chiel, E., Gerling, D., Portnoy, V., Steinberg, S., et al. (2006) Identification and localization of a Rickettsia sp. in Bemisia tabaci (Homoptera: Aleyrodidae). Appl Environ Microbiol 72: 3646-3652.
Gottlieb, Y., Zchori-Fein, E., Mozes-Daube, N., Kontsedalov, S., Skaljac, M., Brumin, M., et al. (2010) The transmission efficiency of tomato yellow leaf curl virus by the whitefly Bemisia tabaci is correlated with the presence of a specific symbiotic bacterium species. J Virol 84: 9310-9317.

Hahn, M.W., De Bie, T., Stajich, J.E., Nguyen, C., and Cristianini, N. (2005) Estimating the tempo and mode of gene family evolution from comparative genomic data. Genome Res 15: 1153-1160.

Himler, A.G., Adachi-Hagimori, T., Bergen, J.E., Kozuch, A., Kelly, S.E., Tabashnik, B.E., et al. (2011) Rapid spread of a bacterial symbiont in an invasive whitefly is driven by fitness benefits and female bias. Science 332: 254-256.

Jain, M., Munoz-Bodnar, A., and Gabriel, D.W. (2017) Concomitant loss of the glyoxalase system and glycolysis makes the uncultured pathogen "Candidatus Liberibacter asiaticus" an energy scavenger. Appl Environ Microbiol 83: e01670-e01617.

Kikuchi, Y., and Fukatsu, T. (2005) Rickettsia infection in natural leech populations. Microb Ecol 49: 265-271.

Kikuchi, Y., Sameshima, S., Kitade, O., Kojima, J., and Fukatsu, T. (2002) Novel clade of Rickettsia spp. from leeches. Appl Environ Microbiol 68: 999-1004.

Kliot, A., Cilia, M., Czosnek, H., and Ghanim, M. (2014) Implication of the bacterial endosymbiont Rickettsia spp. in interactions of the whitefly Bemisia tabaci with tomato yellow leaf curl virus. J Virol 88: 5652-5660.

Konstantinidis, K.T., and Tiedje, J.M. (2005) Genomic insights that advance the species definition for prokaryotes. PNAS 102: 2567-2572.

Küchler, S.M., Kehl, S., and Dettner, K. (2009) Characterization and localization of Rickettsia sp. in water beetles of genus Deronectes (Coleoptera: Dytiscidae). FEMS Microbiol Ecol 68: 201-211.

Lanfear, R., Frandsen, P.B., Wright, A.M., Senfeld, T., and Calcott, B. (2016) PartitionFinder 2: new methods for selecting partitioned models of evolution for molecular and morphological phylogenetic analyses. Mol Biol Evol 34: 772-773.

Li, L., Stoeckert, C.J., and Roos, D.S. (2003) OrthoMCL: identification of ortholog groups for eukaryotic genomes. Genome Res 13: 2178-2189.

Li, T., Xiao, J.H., Xu, Z.H., Murphy, R.W., and Huang, D.W. (2011) Cellular tropism, population dynamics, host range and taxonomic status of an aphid secondary symbiont, SMLS (Sitobion miscanthi L type symbiont). PLoS One 6: e21944.

Liu, S.S., Colvin, J., and De Barro, P.J. (2012) Species concepts as applied to the whitefly Bemisia tabaci systematics: how many species are there? J Integr Agr 11: 176-186.

Liu, S.S., De Barro, P.J., Xu, J., Luan, J.B., Zang, L.S., Ruan, Y.M., and Wan, F.H. (2007) Asymmetric mating interactions drive widespread invasion and displacement in a whitefly. Science 318: 1769-1772.

Luan, J.B., Shan, H.W., Isermann, P., Huang, J.H., Lammerding, J., Liu, S.S., and Douglas, A.E. (2016) Cellular and molecular remodelling of a host cell for vertical transmission of bacterial symbionts. Proc $R$ Soc $B$ 283: 20160580. 
Machtelinckx, T., Van Leeuwen, T., Van De Wiele, T., Boon, N., De Vos, W.H., Sanchez, J.A., et al. (2012) Microbial community of predatory bugs of the genus Macrolophus (Hemiptera: Miridae). BMC Microbiol 12: 1.

Montllor, C.B., Maxmen, A., and Purcell, A.H. (2002) Facultative bacterial endosymbionts benefit pea aphids Acyrthosiphon pisum under heat stress. Ecol Entomol 27: 189-195.

Nirgianaki, A., Banks, G.K., Frohlich, D.R., Veneti, Z., Braig, H.R., Miller, T.A., et al. (2003) Wolbachia infections of the whitefly Bemisia tabaci. Curr Microbiol 47: 0093-0101.

Noda, H., Watanabe, K., Kawai, S., Yukuhiro, F., Miyoshi, T., Tomizawa, M., et al. (2012) Bacteriomeassociated endosymbionts of the green rice leafhopper Nephotettix cincticeps (Hemiptera: Cicadellidae). Appl Entomol Zool 47: 217-225.

Ogata, H., La Scola, B., Audic, S., Renesto, P., Blanc, G., Robert, C., et al. (2006) Genome sequence of Rickettsia bellii illuminates the role of amoebae in gene exchanges between intracellular pathogens. PLoS Genet 2: e76.

Perotti, M.A., Clarke, H.K., Turner, B.D., and Braig, H.R. (2006) Rickettsia as obligate and mycetomic bacteria. FASEB J 20: 2372-2374.

Pilgrim, J., Ander, M., Garros, C., Baylis, M., Hurst, G.D., and Siozios, S. (2017) Torix group Rickettsia are widespread in Culicoides biting midges (Diptera: Ceratopogonidae), reach high frequency and carry unique genomic features. Environ Microbiol 19: 4238-4255.

Rao, Q., Rollat-Farnier, P.A., Zhu, D.T., Santos-Garcia, D., Silva, F.J., Moya, A., et al. (2015) Genome reduction and potential metabolic complementation of the dual endosymbionts in the whitefly Bemisia tabaci. BMC Genomics 16: 1.

Reeves, W.K., Kato, C.Y., and Gilchriest, T. (2008) Pathogen screening and bionomics of Lutzomyia apache (Diptera: Psychodidae) in Wyoming, USA. J AmMosq Control Assoc 24: 444-447.

Russell, J., Latorre, A., Sabater-Muñoz, B., Moya, A., and Moran, N. (2003) Side-stepping secondary symbionts: widespread horizontal transfer across and beyond the Aphidoidea. Mol Ecol 12: 1061-1075.

Richter, M., and Rosselló-Móra, R. (2009) Shifting the genomic gold standard for the prokaryotic species definition. PNAS 106: 19126-19131.

Ronquist, F., Teslenko, M., van der Mark, P., Ayres, D.L., Darling, A., Höhna, S., et al. (2012) MrBayes 3.2: efficient Bayesian phylogenetic inference and model choice across a large model space. Syst Biol 61: 539-542.

Singh, S.T., Priya, N.G., Kumar, J., Rana, V.S., Ellango, R., Joshi, A., et al. (2012) Diversity and phylogenetic analysis of endosymbiotic bacteria from field caught Bemisia tabaci from different locations of North India based on 16S rDNA library screening. Infect Genet Evol 12: 411-419.

Stincone, A., Prigione, A., Cramer, T., Wamelink, M., Campbell, K., Cheung, E., et al. (2015) The return of metabolism: biochemistry and physiology of the pentose phosphate pathway. Biol Rev 90: 927-963.
Takami, H., Taniguchi, T., Arai, W., Takemoto, K., Moriya, Y., and Goto, S. (2016) An automated system for evaluation of the potential functionome: MAPLE version 2.1. 0. DNA Res 23: 467-475.

Wattam, A.R., Abraham, D., Dalay, O., Disz, T.L., Driscoll, T., Gabbard, J.L., et al. (2014) PATRIC, the bacterial bioinformatics database and analysis resource. Nucleic Acids Res 42: D581-d591.

Weeks, A.R., Velten, R., and Stouthamer, R. (2003) Incidence of a new sex-ratio-distorting endosymbiotic bacterium among arthropods. Pro $R$ Soc $B$ 270: 18571865.

Weinert, L.A., Werren, J.H., Aebi, A., Stone, G.N., and Jiggins, F.M. (2009) Evolution and diversity of Rickettsia bacteria. BMC Biol 7: 1.

Weisburg, W.G., Barns, S.M., Pelletier, D.A., and Lane, D.J. (1991) 16S ribosomal DNA amplification for phylogenetic study. J Bacteriol 173: 697-703.

Will, T., Furch, A.C., and Zimmermann, M.R. (2013) How phloem-feeding insects face the challenge of phloemlocated defenses. Front Plant Sci 4: 336.

Xu, H.X., Qian, L.X., Wang, X.W., Shao, R.X., Hong, Y., Liu, S.S., and Wang, X.W. (2019) A salivary effector enables whitefly to feed on host plants byeliciting salicylic acid-signaling pathway. PNAS 116: 490-495.

Zchori-Fein, E., and Brown, J.K. (2002) Diversity of prokaryotes associated with Bemisia tabaci (Gennadius) (Hemiptera: Aleyrodidae). Ann Entomol Soc Am 95: 711-718.

\section{Supporting Information}

Additional Supporting Information may be found in the online version of this article at the publisher's web-site:

Fig. S1 Negative control of FISH in different organs of MED. Fish images: (A) Egg, (B) Fourth instar nymph, (C) Male adult, (D) Female adult, (E) Bacteriocyte, (F) Midgut, (G) Salivary gland, $(\mathrm{H})$ Ovary, (I) Testis. Scale bar is $100 \mu \mathrm{m}$ for Figs. A, B, C, and $20 \mu \mathrm{m}$ for Figs. D, E, F, G.

Fig. S2 COG-based characterization of all proteins with annotated functions in RiTBt.

Fig. S3 Venn diagram presenting the orthologous/ paralogous gene clusters among a range of Rickettsia strains.

Fig. S4 Heat map of the protein diversity profiles of 519 core genes in 64 Rickettsia genomes.

Fig. S5 Vectors of Torix Rickettsia.

Table S1 Detection of RiTBt in various $B$. tabaci populations in China.

Table S2 Function annotations of RiTBt genome.

Table S3 P-T4SS and the tra conjugative DNA-transfer gene element in the RiTBt.

Table S4 Comparison of RiTBt and other published Rickettsia genomes.

Table S5 Single-copy nuclear genes for phylogenomic construction. 